\title{
Essential Role of Nonessential Amino Acid Glutamine in Atherosclerotic Cardiovascular Disease
}

\author{
Jinna Chen, ${ }^{1, *}$ Shulei Zhang, ${ }^{1, *}$ Jiaxiong Wu, Shiyuan $\mathrm{Wu}^{2}{ }^{1}$ Gaosheng $\mathrm{Xu},{ }^{2}$ and Dangheng Wei ${ }^{1}$
}

\begin{abstract}
Atherosclerosis is a major disease that seriously harms human health and is known as the "number one killer" in developed countries and the leading cause of death worldwide. Glutamine is the most abundant nonessential amino acid in the human blood that has multifaceted effects on the body. Recent studies showed that glutamine is negatively corrected with the progression of atherosclerotic lesions. In this review, we focused on the relationship of glutamine with macrophage polarization, nitrification stress, oxidative stress injury, myocardial ischemia-reperfusion injury, and therapeutic angiogenesis to review its roles in atherosclerotic cardiovascular disease.
\end{abstract}

Keywords: glutamine, atherosclerosis, macrophages polarization, ischemia-reperfusion, angiogenesis

\section{Introduction}

C ARDIOVASCULAR DISEASE (CVD) IS the disorder of the heart and blood vessels including the coronary artery, kidney, and cerebral circulation, which is characterized by acute and chronic ischemic syndromes, such as acute coronary syndrome (ACS), angina pectoris, claudication, ischemic stroke, congestive heart failure, and sudden and nonsudden cardiac death (Libby et al., 2018; Partida et al., 2018). CVD is currently the number one killer in the society (Joseph et al., 2017). In the past decades, identifying and reversing the pathogenesis of coronary heart disease (CAD) and preventing its complications remain a major challenge for CAD clinical management (Koton et al., 2014).

Atherosclerosis (As) is the major cause of CAD (Rizzacasa et al., 2019). It refers to the loss of elasticity of arteries and the accumulation of lipid and fibrous material in the arterial wall, narrowing of the arteries for the formation of atherosclerotic plaques (Wolf and Ley, 2019). With the progression of atherosclerotic lesions, plaques in the coronary arteries may block blood flow to the heart. The complete blockage of coronary artery and thrombus induced by plaque rupture can cause a heart attack and lead to massive cell death in the heart (Rigatelli et al., 2019).

The pathophysiology of As is complicated. Recent studies revealed that amino acids are involved in the development of atherosclerotic lesions (Rom et al., 2017). Glutamine is the most abundant free amino acid in the human body (Huang et al., 2017; Kim and Kim, 2017a). Li et al. (2018) showed that the knockout of P-selectin glycoprotein ligand1 inhibits the initiation and progression of atherosclerotic lesions in low-density lipoprotein receptor $\left(L D L R^{-/}\right)$mice accompanied by the changes in amino acid metabolism, which is characterized by increased glycine, valine, alanine, and glutamine. These results showed that glutamine is involved in the initiation and progression of atherosclerotic lesions. In this review, we present an overview of current evidence and functional roles of glutamine in atherosclerotic CVD and discuss the molecular pathways involved in atherosclerotic cardiovascular system.

\section{The Resource and Function of Glutamine}

Glutamine is considered as a conditional essential amino acid, which is synthesized by glutamic acid and ammonia through glutamine synthetase under normal physiological conditions (Wise and Thompson, 2010; Wu et al., 2011). This molecule is the most abundant free amino acid and accounts for $\sim 50 \%$ of the total free amino acids in the human body (Spodenkiewicz et al., 2016). Diet is the most important source of glutamine (Labow and Souba, 2000). The normal fasting plasma glutamine concentration is 500$750 \mu \mathrm{M}$ (Altman et al., 2016).

Glutamine has multifaceted effects on the body. (1) It provides the body with necessary amount of nitrogen. The

\footnotetext{
${ }^{1}$ Institute of Cardiovascular Disease, Key Laboratory for Arteriosclerology of Hunan Province, Hunan International Scientific and Technological Cooperation Base of Arteriosclerotic Disease, Province Innovative Training Base for Medical Postgraduates, Hengyang Medical College, University of South China, Hengyang, Hunan, China.

${ }^{2}$ YueYang Maternal-Child Medicine Health Hospital Hunan, Province Innovative Training Base for Medical Postgraduates, Yueyang, Hunan, China.

*Contributed equally.
} 
biosynthesis of other amino acids is heavily dependent on glutamine (Timmerman et al., 2013). (2) Glutamine improves exercise endurance and can produce alkali, which prevents the decline of exercise capacity or fatigue caused by acidic substances to a certain extent (Coqueiro et al., 2019). (3) Glutamine has important immunomodulatory effects that are required for lymphocyte secretion, proliferation, and maintenance of its function (Dos Santos et al., 2017). (4) This molecule is involved in the synthesis of glutathione (GSH), which exerts strong antioxidative effect against stress damage (Hartmann et al., 2017). (5) Glutamine is an essential nutrient for the metabolism of intestinal mucosal cells and maintains the integrity of the intestinal mucosal epithelial structure (Kim and Kim, 2017a). Under severe stress, such as trauma, infection, and fatigue, glutamine is rapidly depleted in the intestinal mucosal epithelial cells (Wang et al., 2017). (6) This molecule is the substrate of the synthesis of proteins, lipids, and other macromolecular organisms and hence plays important roles in cells growth and proliferation (Fig. 1) (Showalter et al., 2017; Ward, 2010; Son et al., 2013; Bhutia et al., 2015; Altman et al., 2016; Mills et al., 2016; Spinelli et al., 2017).

Glutamine is heavily consumed and used for energy generation as carbon and nitrogen sources for biomass accumulation in rapidly dividing cells, such as lymphocytes, intestinal cells of the small intestine, and especially cancer cells (Zhang et al., 2017). In this process, the glutamine requirement exceeds cellular production, and the cell must increase its intake of this molecule. Moreover, GSH con- sumption is important in situations where catabolism is stressed, such as after surgery, injury, or sepsis (Singleton et al., 2005; Lai et al., 2014; Shu et al., 2016).

\section{Glutamine Promotes the Polarization of M2 Macrophages}

Coronary plaque rupture and thrombosis are the most important pathological bases of ACS, which endangers patients' life with CAD (Bentzon et al., 2014). Rupture of unstable plaque can lead to myocardial infarction (MI), which is the main cause of onset and death of ACS (Badimon and Vilahur, 2014).

The differentiation and activation of monocytes or macrophages are the key processes for the development and stability of As (Cochain and Zernecke, 2015). During this illness, monocytes migrate from the bloodstream into the subintima and differentiate into macrophages, which then undergo different polarization states after being stimulated by different signals (Kawano et al., 2019; Liu et al., 2019). The activation states of macrophages are divided into M1 (classical activation) and M2 (alternatively activation) polarizations (Murray et al., 2014). In vitro, the activation of macrophages induced by lipopolysaccharide (LPS) + IFN- $\gamma$ is the classical activation. However, the activation of macrophages induced by IL-4 is alternatively activation (Martinez and Gorgon, 2014; Orecchioni et al., 2019). M1 macrophages induce a rapid proinflammatory response to infection and tissue damage by sensing the release of injury-related

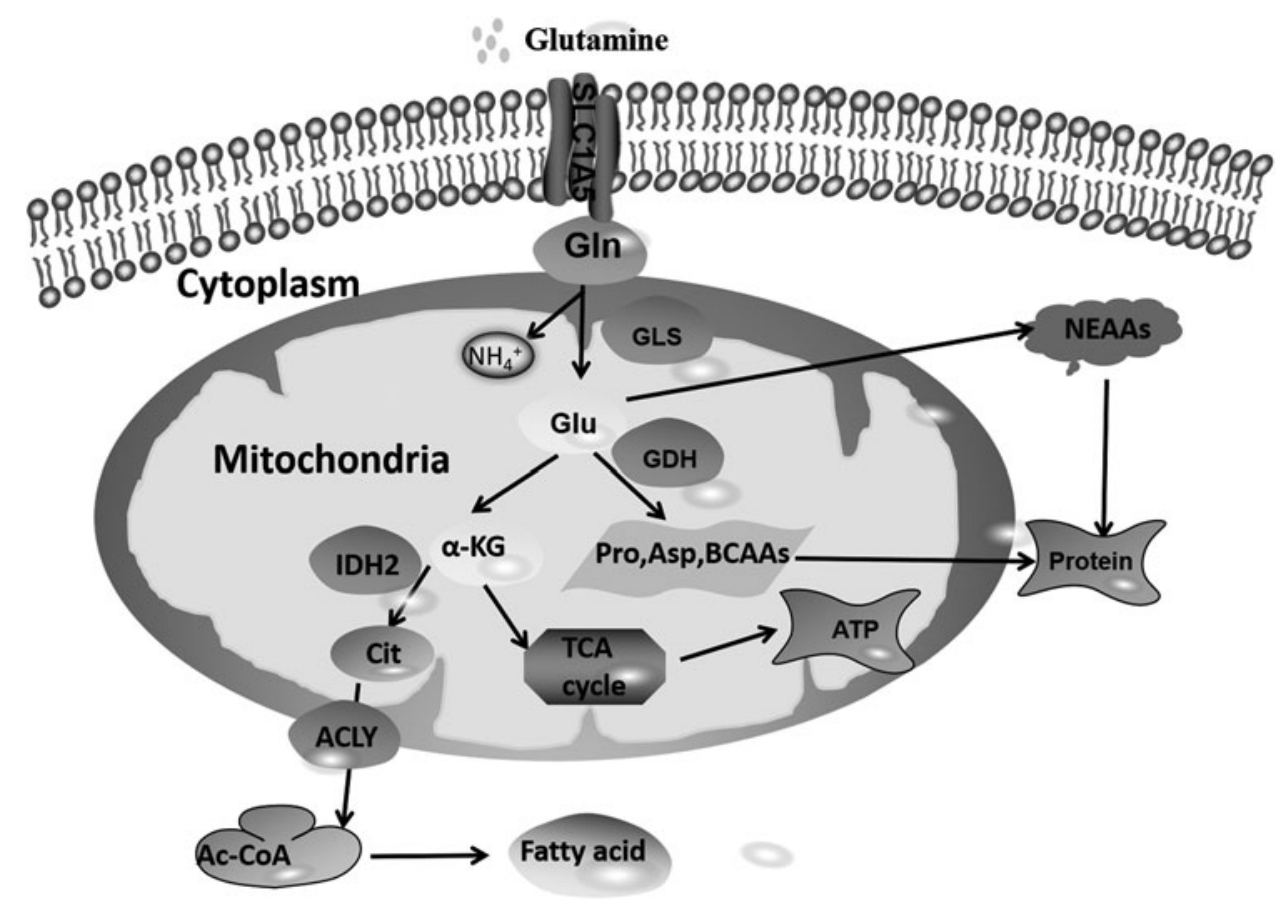

FIG. 1. The important role of glutamine and glutamine-derived glutamate in biosynthesis. Gln, glutamine; SLC1A5, glutamine enters the mammalian cell through transporter (SLC1A5); GLS, glutaminase; GDH, glutamate dehydrogenase; GLDH1, glutamate dehydrogenase $1 ;$ a-KG, $\alpha$-ketoglutaric acid; a-KG enters the TCA cycle to produce ATP; ACLY, ATP citrate lyase; Ac-CoA, acetyl-CoA, glutamine is reduced by carboxylation and reverse carboxylation produces acetyl-CoA to promote fatty acid production; Pro, proline; Asp, aspartic acid; BCAAs, leucine, isoleucine, valine, these amino acids contributes to protein together with nonessential amino acids (NEAAs: glycine, alanine, serine) produced by transaminase by glutamate synthesis. NEAAs, nonessential amino acids; TCA, tricarboxylic acid. 
molecular patterns from microbial components, such as lipopolysaccharides (LPS) and damaged tissues. In contrast, M2 macrophages display anti-inflammatory and repair activities to resolve inflammation and promote tissue repair (Ivashkiv, 2013; Murray et al., 2014). In general, M1 macrophages promote the progression of atherosclerotic plaque and its instability, whereas M2 macrophages inhibit the progression of atherosclerotic plaque and contribute to the stability of plaque (Jia et al., 2017).

Glutamine accumulation leads to macrophage polarization (Jha et al., 2015). A high glutamine uptake by the macrophage indicates a high anti-inflammatory macrophage population (Tavakoli et al., 2017). Glutamine can affect M2 macrophage polarization through uridine $5^{\prime}$-diphosphate- $N$ acetylglucosamine biosynthesis and $N$-glycosylation (Jha et al., 2015). Deprivation or inhibition of $N$-glycosylation by glutamine reduces the polarization of M2 macrophages and the production of $\mathrm{C}$-C motif chemokine ligand 22 (CCL22) (Jha et al., 2015). One-third of the carbon source and $>50 \%$ of the nitrogen in the tricarboxylic acid (TCA) metabolites of M2 macrophages are derived from glutamine (Jha et al., 2015). After the removal of this molecule, the expression of the specific markers of M2 macrophages, including interferon regulatory factor 4 (IRF4), kinesin family member 4 (KIF4), CCL2, and interleukin 4-induced 1 (ILAil), and the TCA cycling activity are substantially downregulated. However, this regulation has no remarkable effect on the polarization of M1 macrophages (Jha et al., 2015). Liu et al. (2017b) revealed that during glutamine deprivation, the specific markers of M1 macrophages (including $I L-1 b, T N F-\alpha, I L-6$, and $I L-12)$ are upregulated, whereas those of M2 macrophages (including Arg1, Ym1 [Chil3], Retnla, and Mrcl) are downregulated through $\alpha \mathrm{KG}$-jmjd3 pathway (Fig. 2). Jha et al. (2015) showed that one-third of all the carbon in TCA metabolites in M2 cells came from glutamine, and more than half of the nitrogen in UDP-GlcNAc synthesis came from glutamine, which also confirmed that this molecule plays a key role in M2 polarization .

These results showed that glutamine plays an important regulatory role and promotes the polarization of M2 macrophages, thus inhibiting the progression of atherosclerotic lesions and benefitting the stability of atherosclerotic plaques.

\section{Glutamine Inhibits Nitrification Stress Caused by Excessive Nitric Oxide}

Nitric oxide (NO) is produced by NO synthase (NOS), a family composed of neuronal NOS (nNOS), inducible NOS (iNOS), and endothelial NOS (eNOS) (Iwakiri and Kim, 2015). In this synthesis process, nicotinamide-adenine dinucleotide phosphate (NADPH), tetrahydrobiopterin, $\mathrm{Ca}^{+2} /$ calmodolin, and flavin adenine dinucleotide act as cofactors (Hong et al., 2019). The most important roles of NO is as an endodermal vasodilator that is critical for cardiovascular protection (Ramirez-Carracedo et al., 2018). NO also inhibits platelet aggregation and adhesion, which contributes to the downregulation of chemotactic protein MCP-1 and surface adhesion molecules, such as $C D 11 / C D 18$, P-selectin, intercellular cell adhesion molecule-1, and vascular cell adhesion molecule-1 (Saini et al., 2012). However, under abnormal conditions such as hypercholesterolemic and portal hypertension, the excessive NO reacts with superoxide and leads to the formation of nitrous oxide or proximities (ONOO); this phenomenon is known as nitrification stress and has serious toxic effects on islet B cells and intermuscular plexus neuron cells (Halliwell, 2006; Terra et al., 2012; Yuste et al., 2015).

The excessive NO activates mitochondrial apoptosis through a variety of pathways, thereby inducing oxidative stress and nitrifying stress, which is closely related to the formation of ONOO by superoxide reactions during the late stages of As (Isshiki et al., 2002). In addition, the combination of NO with the increased superoxide production leads to the formation of harmful proximities, which indirectly (through free radical-mediated reactions) or directly (through oxidation reactions) induces lipid peroxidation and affects cell components and accelerates the formation of atherosclerotic lesions (Rizzo et al., 2003). Consistent with these findings, Shi et al. (2002) showed that high NO concentration aggravates late-stage atherosclerotic lesions by modifying low-density lipoprotein (LDL) oxidation (Shi et al., 2002). In the intermuscular plexus of tumor-bearing rats, NO-mediated nitrification stress injury was alleviated by glutamine (Vicentini et al., 2017).

Endothelial cells (ECs) can synthesize glucosamine from glutamine and glucose. Glucosamine reduces the cellular
FIG. 2. Glutamine affects macrophage polarization. Glutamine deprivation upregulated the expression of M1-type macrophage marker genes. Glutamine promotes the polarization of M2-type macrophages through the akg-jmjd3 pathway, and the expression of M2-type macrophages-related genes are downregulated after the removal of glutamine.

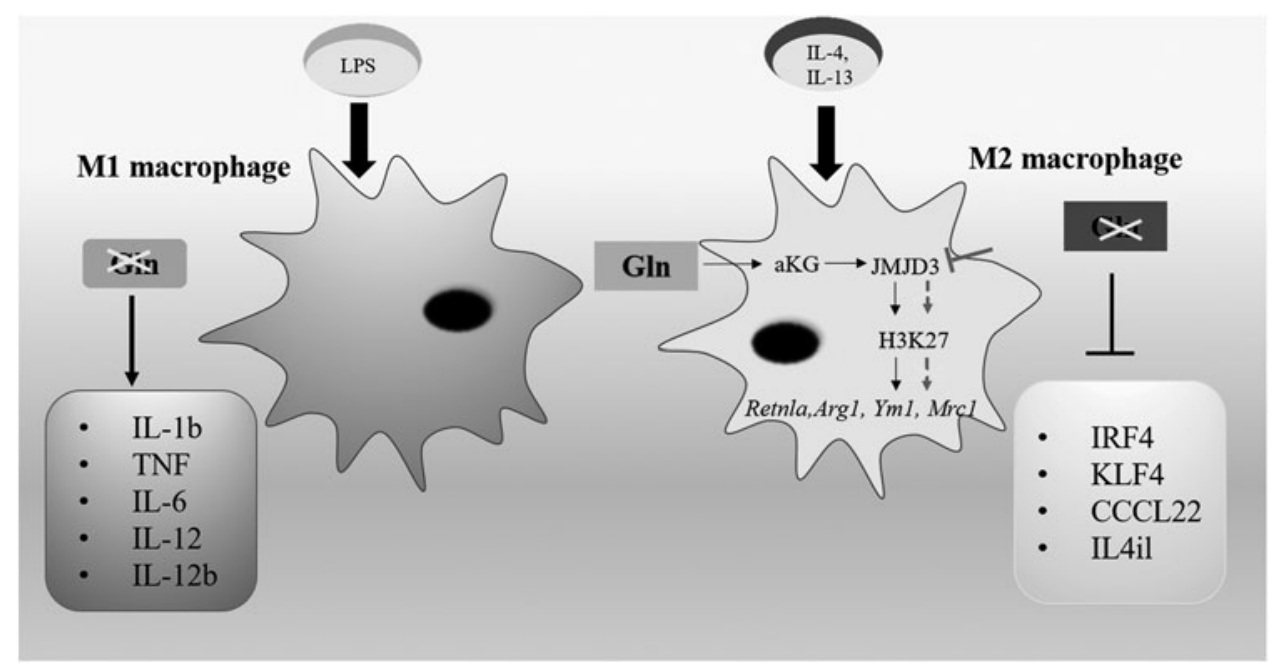


level of NOS cofactor NADPH, which mediates the inhibitory effect of glutamine on endothelial NO synthesis. Beyond this process, glutamine involves in the conversion to glucosamine in hexosamine biosynthetic pathway, thereby inhibiting the activity of oxidative pentose phosphate pathway and reducing the availability of NADPH to inhibit NO synthesis (Guoyao et al., 2001). Besides, glutamine inhibits the accumulation of ammonia-induced striatum cyclicguanosine monophosphate and limits the supply of arginine for NO synthesis (Hilgier et al., 2009). In addition, Gong et al. (2017) showed that oral glutamine with probiotics remarkably reduces NO content by downregulating the expression of iNOS through increasing methylation of the iNOS gene, thereby reducing the expression of the inflammatory factors $T N F-\alpha, I L-6$, and $I L-8$, which contributes to the inhibition of intestinal inflammation and oxidative stress in Wistar rat burn models. The deletion of iNOS promotes cholesterol efflux through increasing the expression of liver $\mathrm{X}$ receptor $\alpha$, ATP-binding cassette transporter $\mathrm{A} 1$, and scavenger receptor class B type I, which retards the progression of As in apoE ${ }^{-/-}$mice (Zhao et al., 2014).

\section{Glutamine Blocks Oxidative Stress Injury Induced by Reactive Oxygen Species}

Oxidative stress is characterized by an imbalance between the production and elimination of cellular oxidants, which damage the basic components of cells (including proteins, lipids, and DNA), interfere with homeostasis, and lead to various adverse consequences (Schieber and Chandel, 2014; $\mathrm{Wu}$ and $\mathrm{Ni}, 2015)$. Oxidative stress induces vascular endothelial cell (VEC) injury and is the main factor of As (Forstermann et al., 2017). Atherosclerotic lesion is a high oxidative stress state characterized by the accumulation of oxidized of lipids and proteins in blood vessel walls (Sun et al., 2013). Reactive oxygen species (ROS), a marker of oxidative stress, is mainly produced in the mitochondrial electron transport chain during cell metabolism and plays important roles in cell biological behaviors, such as proliferation and apoptosis (Cameron et al., 2019). Recent studies showed that ROS increases the pyroptosis of VEC that contributes to VEC dysfunction and As progression (Wu et al., 2018; Zhaolin et al., 2019). Lowering oxidative stress injury induced by ROS is considered as a promising therapy to inhibit the progression of As (Burtenshaw et al., 2019).

GSH is a major endogenous cellular antioxidant that plays an important role in cellular responses to oxidative stress by neutralizing free radicals and ROS. Glutamine produces GSH and GSSG "buffers" of oxidative stress (Ballatori et al., 2009). Decreasing glutaminase and consequently inhibiting glutamine production can downregulate this important antioxidant pathway, leading to high ROS levels and toxic effects on cells (Abu et al., 2017). Glutamine starvation has been reported to deplete endogenous levels of the antioxidant GSH, promote oxidative stress in $\mathrm{HuH}-7$ cells, and induce apoptosis (Yang et al., 1998). ECs are highly susceptible to ROS-induced damage because of glutamine consumption (Huang et al., 2017). In addition, glutaminestarved ECs are more sensitive to ROS-induced cell death (Huang et al., 2017). Similarly, glutamine-depleted ECs show a tendency to decrease the overall level of GSH (Huang et al., 2017). Thus, glutamine supplementation re- duces mitochondrial ROS formation and apoptosis induced by high glucose and hypoxia reoxygenation by decreasing oxidative stress and inactivating intrinsic apoptosis pathways. This process is mainly dependent on the enhanced GSH synthesis (Li et al., 2015).

\section{Glutamine Improves Myocardial Ischemia-Reperfusion Injury with Antioxidative Stress}

Ischemic heart disease is the leading cause of death worldwide (Mozaffarian et al., 2015). Myocardial ischemia, known as MI, is mostly caused by As and usually causes a large number of cardiac cell death. At present, myocardial ischemia-reperfusion strategies, such as thrombolytic therapy or direct percutaneous coronary intervention, remain the standard and most effective treatment for acute MI because of the limited regenerative capacity of the heart (Laflamme and Murry, 2011). However, the process of myocardial reperfusion can itself induce further cardiomyocyte death, a phenomenon known as myocardial reperfusion injury (I/R) (Hausenloy and Yellon, 2013). I/R injury is mainly caused by excessive oxygen free radicals that are generated during reperfusion and trigger strong proinflammatory response, leading to further myocardial damage (Zhou et al., 2015).

Oxidative stress is the main mechanism leading to myocardial I/R injury. Hence, antioxidative stress is an important strategy against such injury. GSH is an important endogenous antioxidant that has a prominent contribution against oxidative stress (Hartmann et al., 2017). Lian et al. (2018) showed that glutamine improves the oxidative stress response induced by myocardial I/R by enhancing $\mathrm{GSH}$ synthesis. The study reported that the glutamine metabolism in rat heart is inhibited by $\mathrm{I} / \mathrm{R}$, and pretreatment with glutamine reduces $\mathrm{I} / \mathrm{R}$ injury and $\mathrm{MI}$ area with $\mathrm{I} / \mathrm{R}$ injury. MicroRNA-200c (miR-200c) aggravates cardiac I/R injury, whereas miR-200c directly targets glutamine to inhibit glutamine metabolism and promote intracellular ROS accumulation (Liu et al., 2017a). Khogali et al. (1998) showed that glutamine enhances the recovery of acute ischemia in isolated rat hearts in a dose-dependent manner. Zhang et al. (2011) found that isolated rat hearts with I/R injury treated with glutamine resulted in the complete recovery of the cardiac output. Glutamine enhances heat shock protein 72 expression, which attenuates intracellular oxidant production, protects cardiomyocytes from I/R damage, and remarkably increases myocardial cell survival and recoveries of systolic function after I/R injury (Zhang et al., 2011). These results showed that glutamine protects against I/R injury through the antioxidant pathway.

\section{Glutamine Improves MI by Promoting Therapeutic Angiogenesis}

Acute MI is a serious ischemic disease. With the introduction of new drugs and the improvement of treatment regimens, the short- and long-term mortality rates of acute MI have been remarkably reduced. The promotion of angiogenesis in ischemic regions has been recently used to treat ischemic diseases and may provide new treatment for CVDs (Kumagai et al., 2018; Lee et al., 2019).

EC metabolism is a regulator of angiogenesis (Huang et al., 2017). Glutamine is the most consumed amino acid by 


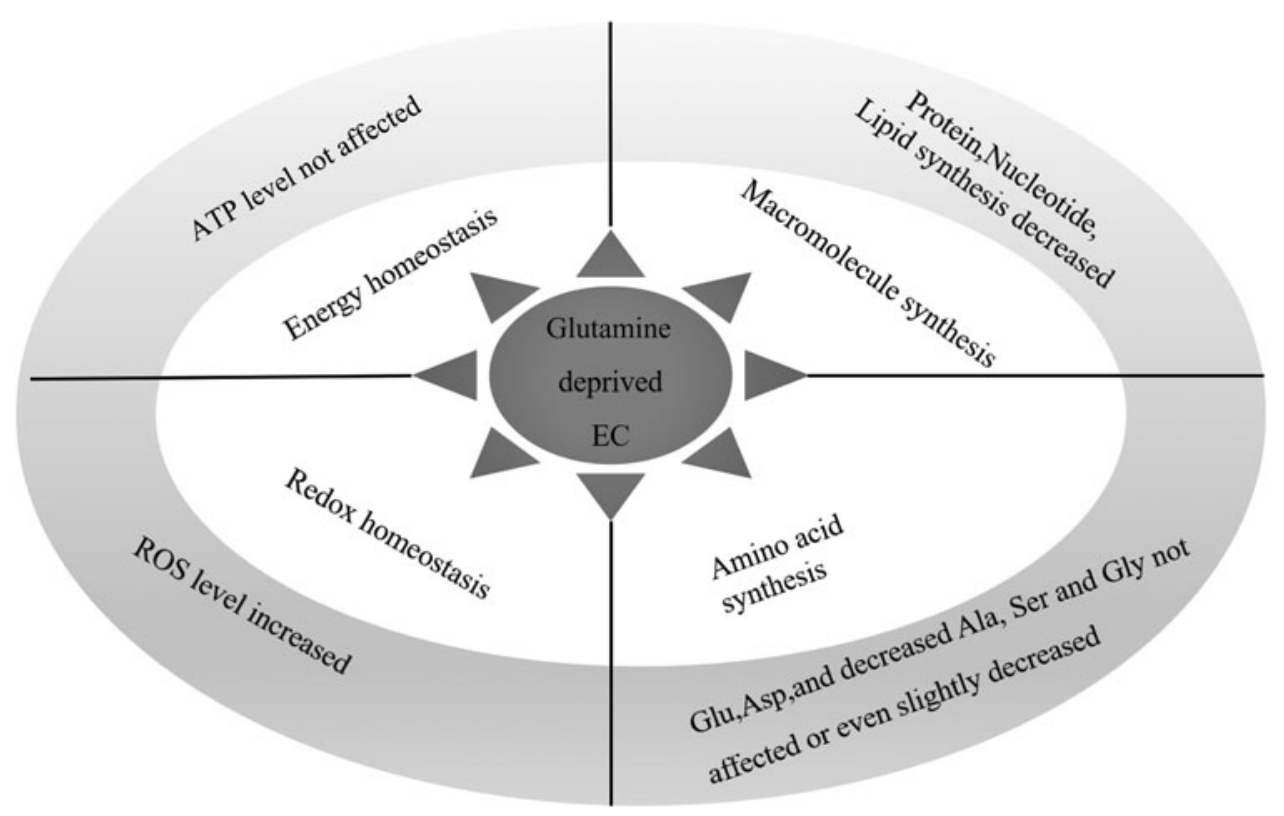

FIG. 3. Glutamine controls various metabolic functions in EC. ECs are a single layer of flat epithelial cells on the surface of blood vessels that come into direct contact with blood. Endothelial dysfunction was found to be the initiating factor of atherosclerosis. (1) Glutamine deprivation depletes the intracellular TCA metabolite pool but does not change the intracellular ATP level. Therefore, glutamine deprivation does not cause an energy crisis, possibly because EC produces most of the energy through glycolysis. (2) Glutamine starvation reduced the storage of glutamate (Glu), aspartic acid (Asp) and asparagine (Asn) in ECS, but did not affect the expression levels of alanine (Ala), serine (Ser) and glycine (Gly). (3) Glutamine deprivation reduces protein, nucleotide, and lipid synthesis. (4) Glutamine deprivation increased intracellular ROS levels and decreased glutathione expression. ECs, endothelial cells; ROS, reactive oxygen species.

vasodilation (Fig. 3) (Kim et al., 2017b). Deprivation of glutamine in ECs or inhibition of glutaminase 1 (GLS1) leads to defects in vessel germination because of impaired proliferation and migration, indicating that glutamine metabolism is essential for EC to reach the tip of the vessel bud (Huang et al., 2017; Kim et al., 2017b). Asparagine is a downstream metabolite of glutamine; inhibition of asparagine GLS1 and deprivation of asparagine with glutamine are lethal for ECs (Li et al., 2017). Supplementation with asparagine can promote the proliferation of ECs after glutamine deprivation. The combination of a-KG and nonessential amino acids (NEAAs) completely rescues the proliferation defects of glutamine-deficient EC. Asparagine is the only NEAA needed for complete recovery. Consistent with its proliferation-rescue effect, the a-KG plus asparagine combination restores intracellular dNTP and rescues all other glutamine-dependent phenotypes, including mTORC1 signaling, protein synthesis, ER stress response, and REDOX imbalances (Fig. 3). These data highlight the importance of asparagine and a-KG supplementation for the metabolism and cellular changes of glutamine-deprived ECs (Huang et al., 2017).

\section{Conclusions and Perspectives}

Glutamine regulates macrophage polarization, oxidative stress, nitrification stress, and angiogenesis and might contribute to the delayed progression of atherosclerotic lesions and the prevention of MI injury. However, some adverse effects of glutamine on CAD are also reported. Rom et al.
(2017) showed that glutamine promotes the accumulation of macrophage triglyceride by enhancing the uptake of LDL and very LDL. Shah et al. (2010) showed that peripheral blood glutamine levels in patients with CAD are higher than that in healthy individuals. Therefore, deeper understanding of the relationship between glutamine and As is necessary to clarify the role of this molecule in the development of atherosclerotic lesions and plaque stability. Moreover, investigation of the underlying mechanism of glutamine in atherosclerotic CVDs will help develop novel strategies for the prevention and treatment of such diseases.

\section{Disclosure Statement}

No competing financial interests exist.

\section{Funding Information}

This work was supported by the grants from the National Natural Science Foundation of China (81370378), the Research project of graduate students in Hunan Province, China, 2017 (CX2017B551).

\section{References}

Abu, A.O., Habib, S.L., Trott, J., Stewart, B., Liang, S., Chaudhari, A.J., et al. (2017). Glutamine addiction in kidney cancer suppresses oxidative stress and can be exploited for real-time imaging. Cancer Res 77, 6746-6758.

Altman, B.J., Stine, Z.E., and Dang, C.V. (2016). From Krebs to clinic: glutamine metabolism to cancer therapy. Nat Rev Cancer 16, 619-634. 
Badimon, L., and Vilahur, G. (2014). Thrombosis formation on atherosclerotic lesions and plaque rupture. J Intern Med 276, 618-632.

Ballatori, N., Krance, S.M., Notenboom, S., Shi, S., Tieu, K., and Hammond, C.L. (2009). Glutathione dysregulation and the etiology and progression of human diseases. Biol Chem 390, 191-214.

Bentzon, J.F., Otsuka, F., Virmani, R., and Falk, E. (2014). Mechanisms of plaque formation and rupture. Circ Res 114, 1852-1866.

Bhutia, Y.D., Babu, E., Ramachandran, S., and Ganapathy, V. (2015). Amino acid transporters in cancer and their relevance to "glutamine addiction": novel targets for the design of a new class of anticancer drugs. Cancer Res 75, 17821788.

Burtenshaw, D., Kitching, M., Redmond, E.M., Megson, I.L., and Cahill, P.A. (2019), reactive oxygen species (ROS), intimal thickening, and subclinical atherosclerotic disease. Front Cardiovasc Med 6, 89.

Cameron, A.M., Castoldi, A., Sanin, D.E., Flachsmann, L.J., Field, C.S., Puleston, D.J., et al. (2019). Inflammatory macrophage dependence on $\mathrm{NAD}(+)$ salvage is a consequence of reactive oxygen species-mediated DNA damage. Nat Immunol 20, 420-432.

Cochain, C., and Zernecke, A. (2015). Macrophages and immune cells in atherosclerosis: recent advances and novel concepts. Basic Res Cardiol 110, 34.

Coqueiro, A.Y.R., Rogero, M.M., and Tirapegui, J. (2019). Glutamine as an anti-fatigue amino acid in sports nutrition. Nutrients 11, pii: E863.

Dos Santos, G.G., Hastreiter, A.A., Sartori, T., Borelli, P., and Fock, R.A. (2017). L-glutamine in vitro modulates some immunomodulatory properties of bone marrow mesenchymal stem cells. Stem Cell Rev 13, 482-490.

Forstermann, U., Xia, N., and Li, H. (2017). Roles of vascular oxidative stress and nitric oxide in the pathogenesis of atherosclerosis. Circ Res 120, 713-735.

Gong, Z.Y., Yuan, Z.Q., Dong, Z.W., and Peng, Y.Z. (2017). Glutamine with probiotics attenuates intestinal inflammation and oxidative stress in a rat burn injury model through altered iNOS gene aberrant methylation. Am J Transl Res 5, 25352547.

Guoyao, W., Haynes, T.E., Li, H., Yan, W., and Meininger, C.J. (2001). Glutamine metabolism to glucosamine is necessary for glutamine inhibition of endothelial nitric oxide synthesis. Biochem J 335, 245-252.

Halliwell, B. (2006). Reactive species and antioxidants. Redox biology is a fundamental theme of aerobic life. Plant Physiol 141, 312-322.

Hartmann, R., Licks, F., Schemitt, E.G., Colares, J.R., Da Silva, J., Moura, R.M.B., et al. (2017). Effect of glutamine on liver injuries induced by intestinal ischemia-reperfusion in rats. Nutr Hosp 34, 548-554.

Hausenloy, D.J., and Yellon, D.M. (2013). Myocardial ischemia-reperfusion injury: a neglected therapeutic target. J Clin Invest 123, 92-100.

Hilgier, W., Fresko, I., Klemenska, E., Beresewicz, A., Oja, S.S., Saransaari, P., et al. (2009). Glutamine inhibits ammonia-induced accumulation of cGMP in rat striatum limiting arginine supply for NO synthesis. Neurobiol Dis 35, $75-81$.

Hong, FF., Liang, X.Y., Liu, W., Lv, S., He, S.J., Kuang, H.B., et al. (2019). Roles of eNOS in atherosclerosis treatment. Inflamm Res 6, 429-441.
Huang, H., Vandekeere, S., Kalucka, J., Bierhansl, L., Zecchin, A., Bruning, U., et al. (2017). Role of glutamine and interlinked asparagine metabolism in vessel formation. Embo J 36, 2334-2352.

Isshiki, M., Ying, Y.S., Fujita, T., and Anderson, R.G. (2002). A molecular sensor detects signal transduction from caveolae in living cells. J Biol Chem 277, 43389-43398.

Ivashkiv, L.B. (2013). Epigenetic regulation of macrophage polarization and function. Trends Immunol 34, 216-223.

Iwakiri, Y., and Kim, M.Y. (2015). Nitric oxide in liver diseases. Trends Pharmacol Sci 36, 524-536.

Jha, A.K., Huang, S.C., Sergushichev, A., Lampropoulou, V., Ivanova, Y., Loginicheva, E., et al. (2015). Network integration of parallel metabolic and transcriptional data reveals metabolic modules that regulate macrophage polarization. Immunity 42, 419-430.

Jia, S.J., Gao, K.Q., and Zhao, M. (2017). Epigenetic regulation in monocyte/macrophage: a key player during atherosclerosis. Cardiovasc Ther 35, e12262.

Joseph, P., Leong, D., McKee, M., Anand, S.S., Schwalm, J.D., Teo, K., et al. (2017). Reducing the global burden of cardiovascular disease, part 1: the epidemiology and risk factors. Circ Res 121, 677-694.

Kawano, A., Ariyoshi, W., Yoshioka, Y., Hikiji, H., Nishihara, T., and Okinaga, T. (2019). Docosahexaenoic acid enhances M2 macrophage polarization via the p38 signaling pathway and autophagy. J Cell Biochem 120, 12604-12617.

Khogali, S.E., Harper, A.A., Lyall, J.A., and Rennie, M.J. (1998). Effects of L-glutamine on post-ischaemic cardiac function: protection and rescue. J Mol Cell Cardiol 30, 819-827.

Kim, B., Li, J., Jang, C., and Arany, Z. (2017b). Glutamine fuels proliferation but not migration of endothelial cells. Embo J 36, 2321-2333.

Kim, M.H., and Kim, H. (2017a). The roles of glutamine in the intestine and its implication in intestinal diseases. Int $\mathrm{J}$ Mol Sci 18, 2-15.

Koton, S., Schneider, A.L., Rosamond, W.D., Shahar, E., Sang, Y., Gottesman, R.F., et al. (2014). Stroke incidence and mortality trends in US communities, 1987 to 2011. JAMA 312, 259-268.

Kumagai, M., Minakata, K., Masumoto, H., Yamamoto, M., Yonezawa, A., Ikeda, T., et al. (2018). A therapeutic angiogenesis of sustained release of basic fibroblast growth factor using biodegradable gelatin hydrogel sheets in a canine chronic myocardial infarction model. Heart Vessels 33, 1251-1257.

Labow, B.I., and Souba, W.W. (2000). Glutamine. World J Surg 24, 1503-1513.

Laflamme, M.A., and Murry, C.E. (2011). Heart regeneration. Nature 473, 326-335.

Lai, C.C., Liu, W.L., and Chen, C.M. (2014). Glutamine attenuates acute lung injury caused by acid aspiration. Nutrients 6, 3101-3116.

Lee, D.J., Cavasin, M.A., Rocker, A.J., Soranno, D.E., Meng, X., Shandas, R., et al. (2019). An injectable sulfonated reversible thermal gel for therapeutic angiogenesis to protect cardiac function after a myocardial infarction. J Biol Eng 13, 6.

Li, B., Lu, X., Wang, J., He, X., Gu, Q., Wang, L., et al. (2018). The metabonomics study of P-selectin glycoprotein ligand-1 (PSGL-1) deficiency inhibiting the progression of atherosclerosis in LDLR mice. Int J Biol Sci 14, 36-46.

Li, J., Song, P., Zhu, L., Aziz, N., Zhou, Q., Zhang, Y., et al. (2017). Synthetic lethality of glutaminolysis inhibition, autophagy inactivation and asparagine depletion in colon cancer. Oncotarget 8, 42664-42672. 
Li, K., Cui, Y.C., Zhang, H., Liu, X.P., Zhang, D., Wu, A.L., et al. (2015). Glutamine reduces the apoptosis of H9C2 cells treated with high-glucose and reperfusion through an oxidation-related mechanism. PLoS One 10, e0132402.

Lian, G., Gnanaprakasam, J.R., Wang, T., Wu, R., Chen, X., Liu, L., et al. (2018). Glutathione de novo synthesis but not recycling process coordinates with glutamine catabolism to control redox homeostasis and directs murine $\mathrm{T}$ cell differentiation. Elife 7, pii: e36158.

Libby, P., Loscalzo, J., Ridker, P.M., Farkouh, M.E., Hsue, P.Y., Fuster, V., et al. (2018). Inflammation, immunity, and infection in atherothrombosis: JACC review topic of the week. J Am Coll Cardiol 72, 2071-2081.

Liu, F., Li, Y., and Liu, G. (2017a). MicroRNA-200c exacerbates the ischemia/reperfusion injury of heart through targeting the glutaminase (GLS)-mediated glutamine metabolism. Eur Rev Med Pharmacol Sci 21, 3282-3289.

Liu, L., Liang, L., Liang, H., Wang, M., Lu, B., Xue, M., et al. (2019). Fusobacterium nucleatum aggravates the progression of colitis by regulating M1 macrophage polarization via AKT2 pathway. Front Immunol 10, 1324.

Liu, P.S., Wang, H., Li, X., Chao, T., Teav, T., Chirsten, S., et al. (2017b). A-ketoglutarate orchestrates macrophage activation through metabolic and epigenetic reprogramming. Nat Immunol 18, 985-994.

Martinez, F.O., and Gorgon, S. (2014). The M1 and M2 paradigm of macrophage activation: time for reassessment. F1000Prime Rep 6, 13.

Mills, E.L., Kelly, B., Logan, A., Costa, A.S.H., Varma, M., Bryant, C.E., et al. (2016). Succinate dehydrogenase supports metabolic repurposing of mitochondria to drive inflammatory macrophages. Cell 167, 457-470.e13.

Mozaffarian, D.B., Benjamin, E.J., Go, A.S., Arnett, D.K., and Cushman, M. (2015). Heart disease and stroke statistics-2015 update: a report from the American Heart Association. Circulation 133, e38-e360.

Murray, P.J., Allen, J.E., Biswas, S.K., Fisher, E.A., Gilroy, D.W., Goerdt, S., et al. (2014). Macrophage activation and polarization: nomenclature and experimental guidelines. Immunity 41, 14-20.

Orecchioni, M., Ghosheh, Y., Pramod, A.B., and Ley, K. (2019). Macrophage polarization: different gene signatures in M1 (LPS+) vs. classically and M2 (LPS-) vs. alternatively activated macrophages. Front Immunol 10, 1084.

Partida, R.A., Libby, P., Crea, F., and Jang, I.K. (2018). Plaque erosion: a new in vivo diagnosis and a potential major shift in the management of patients with acute coronary syndromes. Eur Heart J 39, 2070-2076.

Ramirez-Carracedo, R., Tesoro, L., Hernandez, I., Diez-Mata, J., Filice, M., Toro, R., et al. (2018). Non-invasive detection of extracellular matrix metalloproteinase inducer EMMPRIN, a new therapeutic target against atherosclerosis, inhibited by endothelial nitric oxide. Int J Mol Sci 19, pii: E3248.

Rigatelli, G., Zuin, M., Ngo, T.T., Nguyen, H.T., Nanjundappa, A., Talarico, E., et al. (2019). Intracoronary cavitation as a cause of plaque rupture and thrombosis propagation in patients with acute myocardial infarction: a computational study. J Transl Int Med 7, 69-75.

Rizzacasa, B., Amati, F., Romeo, F., Novelli, G., and Mehta, J.L. (2019). Epigenetic modification in coronary atherosclerosis: JACC review topic of the week. J Am Coll Cardiol 74, 1352-1365.

Rizzo, V., Morton, C., DePaola, N., Schnitzer, J.E., and Davies, P.F. (2003). Recruitment of endothelial caveolae into me- chanotransduction pathways by flow conditioning in vitro. Am J Physiol Heart Circ Physiol 285, H1720-H1729.

Rom, O., Grajeda-Iglesias, C., Najjar, M., Abu-Saleh, N., Volkova, N., Dar, D.E., et al. (2017). Atherogenicity of amino acids in the lipid-laden macrophage model system in vitro and in atherosclerotic mice: a key role for triglyceride metabolism. J Nutr Biochem 45, 24-38.

Saini, V., Bhatnagar, M.K., and Bhattacharjee, J. (2012). Endothelial nitric oxide synthase Glu298Asp (G894T) gene polymorphism in coronary artery disease patients with type 2 diabetes mellitus. Diabetes Metab Syndr 6, 106-109.

Schieber, M., and Chandel, N.S. (2014). ROS function in redox signaling and oxidative stress. Curr Biol 24, R453-R462.

Shah, S.H., Bain, J.R., Muehlbauer, M.J., Stevens, R.D., Crosslin, D.R., Haynes, C., et al. (2010). Association of a peripheral blood metabolic profile with coronary artery disease and risk of subsequent cardiovascular events. Circ Cardiovasc Genet 3, 207-214.

Shi, W., Wang, X., Shih, D.M., Laubach, V.E., Navab, M., and Lusis, A.J. (2002). Paradoxical reduction of fatty streak formation in mice lacking endothelial nitric oxide synthase. Circulation 105, 2078-2082.

Showalter, M.R., Hatakeyama, J., Cajka, T., Vandervorst, K., Iii, K.L.C., and Fiehn, O. (2017). Replication study: the common feature of leukemia-associated IDH1 and IDH2 mutations is a neomorphic enzyme activity converting alphaketoglutarate to 2-hydroxyglutarate. Elife 6, pii: e26030.

Shu, X., Zhang, J., Wang, Q., Xu, Z., and Yu, T. (2016). Glutamine decreases intestinal mucosal injury in a rat model of intestinal ischemia-reperfusion by downregulating HMGB1 and inflammatory cytokine expression. Exp Ther Med 12, 1367-1372.

Singleton, K.D., Serkova, N., Beckey, V.E., and Wischmeyer, P.E. (2005). Glutamine attenuates lung injury and improves survival after sepsis: role of enhanced heat shock protein expression. Crit Care Med 33, 1206-1213.

Son, J., Lyssiotis, C.A., Ying, H., Wang, X., Hua, S., Ligorio, M., et al. (2013). Glutamine supports pancreatic cancer growth through a KRAS-regulated metabolic pathway. Nature 496, 101-105.

Spinelli, J.B., Yoon, H., Ringel, A.E., Jeanfavre, S., Clish, C. B., and Haigis, M.C. (2017). Metabolic recycling of ammonia via glutamate dehydrogenase supports breast cancer biomass. Science 358, 941-946.

Spodenkiewicz, M., Diez-Fernandez, C., Rufenacht, V., Gemperle-Britschgi, C., and Haberle, J. (2016). Minireview on glutamine synthetase deficiency, an ultra-rare inborn error of amino acid biosynthesis. Biology (Basel) 5, pii: E40.

Sun, G.B., Qin, M., Ye, J.X., Pan, R.L., Meng, X.B., Wang, M., et al. (2013). Inhibitory effects of myricitrin on oxidative stress-induced endothelial damage and early atherosclerosis in ApoE-/- mice. Toxicol Appl Pharmacol 271, 114-126.

Tavakoli, S., Downs, K., Short, J.D., Nguyen, H.N., Lai, Y., Jerabek, P.A., et al. (2017). Characterization of macrophage polarization states using combined measurement of 2deoxyglucose and glutamine accumulation implications for imaging of atherosclerosis. Arterioscler Thromb Vasc Biol 37, 1840-1848.

Terra, V.A., Souza-Neto, F.P., Pereira, R.C., Silva, T.N., Costa, A.C., Luiz, R.C., et al. (2012). Time-dependent reactive species formation and oxidative stress damage in the skin after UVB irradiation. J Photochem Photobiol B 109, 34-41.

Timmerman, L.A., Holton, T., Yuneva, M., Louie, R.J., Padro, M., Daemen, A., et al. (2013). Glutamine sensitivity analysis 
identifies the $\mathrm{xCT}$ antiporter as a common triple-negative breast tumor therapeutic target. Cancer Cell 24, 450-465.

Vicentini, G.E., Martins, H.A., Fracaro, L., de Souza S.R.G., da Silva Zanoni, K.P., Silva, T.N.X., et al. (2017). Does 1glutamine-supplemented diet extenuate NO-mediated damage on myenteric plexus of Walker 256 tumor-bearing rats? Food Res Int 101, 24-34.

Wang, J., Li, Y., and Qi, Y. (2017). Effect of glutamineenriched nutritional support on intestinal mucosal barrier function, MMP-2, MMP-9 and immune function in patients with advanced gastric cancer during perioperative chemotherapy. Oncol Lett 14, 3606-3610.

Ward, P.S., Patel, J., Wise, D.R., Abdel-Wahab, O., Bryson, D., Bennett, H.A., and Coller, J. (2010). The common feature of leukemia associated IDH1 and IDH2 mutations is a neomorphic enzyme activity converting $\alpha$-ketoglutarate to 2 hydroxyglutarate. Cancer Cell 17, 225-234.

Wise, D.R., and Thompson, C.B. (2010). Glutamine addiction: a new therapeutic target in cancer. Trends Biochem Sci 35, 427-433.

Wolf, D., and Ley, K. (2019). Immunity and inflammation in atherosclerosis. Circ Res 124, 315-327.

Wu, G., Bazer, F.W., Johnson, G.A., Knabe, D.A., Burghardt, R.C., Spencer, T.E., et al. (2011). Triennial growth symposium: important roles for-glutamine in swine nutrition and production. J Anim Sci 89, 2017-2030.

Wu, Q., and Ni, X. (2015). ROS-mediated DNA methylation pattern alterations in carcinogenesis. Curr Drug Targets 16, $13-19$.

Wu, X., Zhang, H., Qi, W., Zhang, Y., Li, J., Li, Z., et al. (2018). Nicotine promotes atherosclerosis via ROS-NLRP3mediated endothelial cell pyroptosis. Cell Death Dis 9, 171.

Xu, Y., Nguyen, Q., Lo, D.C., and Czaja, M.J. (1998). c-mycdependent hepatoma cell apoptosis results from oxidative stress and not a deficiency of growth factors. J Cell Physiol 170, 192-199.

Yuste, J.E., Tarragon, E., Campuzano, C.M., and Ros-Bernal, F. (2015). Implications of glial nitric oxide in neurodegenerative diseases. Front Cell Neurosci 9, 322.

Zhang, J.P., Pavlova N.N., and Thompson, C.B. (2017). Cancer cell metabolism: the essential role of the nonessential amino acid, glutamine. Embo J 36, 1302-1315.
Zhang, W.X., Zhou, L.F., Zhang, L., Bao, L., Wang, C.C., Meng, H.Y., et al. (2011). Protective effects of glutamine preconditioning on ischemia-reperfusion injury in rats. Hepatobiliary Pancreat Dis Int 10, 78-82.

Zhao, J.F., Shyue, S.K., Lin, S.J., Wei, J., and Lee, T.S. (2014). Excess nitric oxide impairs $\operatorname{LXR}(\alpha)$-ABCA1-dependent cholesterol efflux in macrophage foam cells. J Cell Physiol 229, 117-125.

Zhaolin, Z., Jiaojiao, C., Peng, W., Yami, L., Tingting, Z., Jun, T., et al. (2019). OxLDL induces vascular endothelial cell pyroptosis through miR-125a-5p/TET2 pathway. J Cell Physiol 234, 7475-7491.

Zhou, T.C., Chuang, C.C., and Zuo, L. (2015). Molecular characterization of reactive oxygen species in myocardial ischemia-reperfusion injury. Biomed Res Int 2015, 864946.

Address correspondence to: Dangheng Wei, PhD University of South China Hengyang 421001

China

E-mail: weizhonghua99@126.com

Shiyuan $W u, M D$

YueYang Maternal-Child Medicine Health Hospital

Yueyang 414000

China

E-mail:1530369018@qq.com

Gaosheng Xu, MD

YueYang Maternal-Child Medicine Health Hospital

Yueyang 414000

China

E-mail: xugaosheng0414@163.com

Received for publication August 18, 2019; received in revised form October 8, 2019; accepted October 9, 2019. 